\title{
Andy RUDDOCK, Investigating Audiences
}

London, Sage Publications Ltd, 2007, 185 p.

\section{Céline Ségur}

\section{(2) OpenEdition}

1 Journals

\section{Édition électronique}

URL : http://journals.openedition.org/questionsdecommunication/188

DOI : 10.4000/questionsdecommunication. 188

ISSN : 2259-8901

\section{Éditeur}

Presses universitaires de Lorraine

\section{Édition imprimée}

Date de publication : 1 décembre 2009

ISBN : 978-2-8143-0003-3

ISSN : 1633-5961

\section{Référence électronique}

Céline Ségur, «Andy RUDDock, Investigating Audiences », Questions de communication [En ligne], 16।

2009, mis en ligne le 19 janvier 2012, consulté le 22 septembre 2020. URL : http://

journals.openedition.org/questionsdecommunication/188; DOI : https://doi.org/10.4000/

questionsdecommunication.188

\section{Ce document a été généré automatiquement le 22 septembre 2020}

Tous droits réservés 


\section{Andy RUDDOCK, Investigating Audiences}

London, Sage Publications Ltd, 2007, 185 p.

\section{Céline Ségur}

\section{RÉFÉRENCE}

Andy RUDDock, Investigating Audiences, London, Sage Publications Ltd, 2007, 185 p.

1 Andy Ruddock est un chercheur britannique que l'on ne rencontre pas fréquemment dans les bibliographies de publications francophones sur les publics. Pourtant, dans le monde anglo-saxon, cet enseignant de l'université de Monash (Australie) est reconnu pour ses nombreuses enquêtes sur la réception des médias. Familier des Cultural Studies, il a rédigé en 2001 une réflexion critique sur les recherches concernant les publics (Understanding Audiences [Comprendre les publics], London, Sage Publications), une démarche qu'il poursuit en 2007 avec la publication d'Investigating Audiences [Enquêter sur les publics]. De quoi s'agit-il?

2 Précisons d'emblée l'étendue que recouvre la notion «Audiences» dans la langue anglaise, puisque l'ouvrage traite de toutes les formes de publics, pas seulement médiatiques. En passant en revue les principales problématiques afférentes à ce champ, l'auteur propose un programme de recherche articulé autour de trois idées (voir infra); le tout est illustré par quantité de données issues d'enquêtes empiriques récentes, conduites par Andy Ruddock ainsi que par ses pairs. Ces dernières n'ont pas ou peu circulé dans l'Hexagone, ce qui constitue l'un des nombreux apports de cet ouvrage. En effet, si, en tant que lecteur francophone, on retrouve quelques références théoriques familières, notamment sur l'histoire des recherches sur la réception (avec les incontournables écrits de Stuart Hall, Elihu Katz, Karl Lazarsfeld, Janis A. Radway...), la plupart des noms figurant dans la bibliographie ne sont pas ou peu connus. Ce qui signifie que ce livre offre un panorama détaillé de l'actualité des travaux dans le monde 
anglo-saxon, ainsi que des ouvertures théoriques et méthodologiques pour ceux qui souhaiteraient renouveler les perspectives de recherche de ce domaine.

Un premier versant de la thèse défendue par Andy Ruddock porte sur la notion de contexte, au sens de "cadre " goffmanien (frame). Le contexte de réalisation d'une investigation a une incidence sur la nature des données recueillies et la manière dont elles sont exploitées: l'environnement social, historique et culturel de l'enquêteur « inevitably plays a part in the formulation and operationalization of research questions » [joue un rôle dans la formulation et la mise en œuvre des problématiques, de manière inévitable] (p.24). Pour illustrer ce propos, l'auteur prend appui sur l'antagonisme notoire entre tenants des Uses \& Gratifications researchs et Cultural Studies (chapitre 1, pp. 20-22) : à la fin des années 70, les chercheurs britanniques ont reproché aux enquêtes sur les effets limités des médias d'être elles-mêmes limitées et non abouties. Autre élément de critique: les sondages réalisés n'ont pas permis de distinguer les multiples variations dans les processus de décision étudiés (ces processus sont différents selon la nature de la décision qui est à prendre selon Todd Gitlin). Plus récemment, nous apprend Andy Ruddock, Elihu Katz et Elisabeth Noelle-Neumann ont identifié dans la biographie de Paul Lazarsfeld deux éléments qui permettent d'éclairer ces lacunes: l'un d'ordre économique (manque de financements pour poursuivre les recherches), l'autre d'ordre personnel (le parcours de Paul Lazarsfeld révèle une personnalité peu rigoureuse sur le plan méthodologique). Dès lors, les insuffisances pointées ont une valeur heuristique moindre. Cette problématique de la posture du chercheur- qui n'est pourtant pas spécifique au champ de recherche sur les publicssemble préoccuper particulièrement Andy Ruddock. En témoigne le titre du premier chapitre: "Can I write $I$ in an essay?» [Puis-je écrire Je?]. Dans la mesure où le chercheur travaillant sur la réception est parfois amené à susciter des actes de réception, il intervient en amont des données recueillies. Dans le chapitre 4, l'auteur remarque que les chercheurs qui enquêtent sur les fans font souvent eux-mêmes partie du groupe d'amateurs étudié, et ils l'affirment, presque pour justifier le bien-fondé de l'investigation. Par là, ils donnent corps à ce groupe. Ce que le chercheur explique en faisant référence au concept de « communauté imaginée » de Benedict Anderson (p.80). Cette posture n'est pas à bannir, poursuit-il, car elle donne accès à des données qui n'auraient pu être collectées sans cette implication. Mais cette dernière confère une responsabilité au chercheur (p.25): l'enquêteur-fan a connaissance du cadre de références à partir duquel l'acte de réception est produit, ce qui lui permet de rationnaliser la pratique observée.

En effet, la question du contexte de mise en œuvre de l'investigation prend toute son importance dans la mesure où elle croise celle du contexte de réception. La thèse est avancée à partir d'une critique adressée aux partisans de travaux simplement quantitatifs (qui consistent à interpréter des données chiffrées recueillies de manière expérimentale ou non). Selon Andy Ruddock, si l'on ne prend pas en compte le contexte de réception, on risque d'aboutir à de fausses conclusions, puisque l'enquêteur risque de présupposer lui-même les éléments de ce contexte. Prenons l'exemple d'un chercheur qui enquête sur les effets des médias et le rapport à la violence. À cette fin, il analyse des témoignages recueillis sur ce thème en tant qu'actes de réception : la tenue de ces discours ne laisse pas supposer que leurs auteurs aient établi un rapport de cause à effet entre violence et médias. L'analyse des données doit donc intégrer davantage le cadre de pensée construit par l'enquêté (plutôt que celui du chercheur). En ce sens, il convient de s'interroger sur ce que l'acte de réception étudié ne signifie pas autant que 
sur ce qu'il signifie. L'idée est développée dans le chapitre 2 où il est question du public et de son rapport aux News: si l'on veut comprendre les effets de la circulation de l'information médiatique, il faut arrêter de raisonner à la manière d'une équation mathématique, qui consisterait à se demander si l'information reçue correspond ou non à celle qui est émise. Le processus de réception est plus complexe, affirme Andy Ruddock qui confirme sa thèse dans le chapitre $5:$ nature du récepteur et nature du produit reçu forment un cadre dans lequel l'acte de réception prend place. Ainsi ne s'agit-il plus d'isoler le récepteur du produit qu'il reçoit ni de lire des réceptions possibles dans les textes. Dans l'étude des effets des programmes violents et déviants sur les comportements des individus, il convient de définir au préalable ce que signifie un programme violent/déviant pour l'enquêteur ainsi que pour l'enquêté. Car, «studies have shown that audiences do not necessarily view messages of sex and violence as being about sex and violence " [des travaux ont montré que les publics ne considèrent pas forcément les messages contenant du sexe et de la violence comme étant du domaine du sexe et de la violence] (p.103). Il faut donc préalablement s'interroger sur la catégorie de perception d'un message. Pour reprendre un exemple détaillé par l'auteur, la nudité utilisée en publicité choque davantage que celle présentée dans un magazine pornographique. De la même manière, l'enquêteur doit veiller à ne pas pré-attribuer de signification au message (par exemple, "c'est un message à caractère idéologique »), car il risque d'interpréter sa réception en fonction de sa propre lecture («l'individu adhère ou non à cette idéologie»), alors que le récepteur aura attribué un tout autre sens au contenu- par exemple, il en fait une lecture émotionnelle (chapitre 1, pp. 25-27).

5 Ce qui nous conduit à traiter le troisième aspect du programme défendu par Andy Ruddoc: les médias ne jouent pas un rôle à ce point central dans la vie des individus. Leur influence n'est pas autonome: elle s'exerce en parallèle à d'autres facteurs d'influence (voir le contexte). Le chercheur doit donc s'immuniser contre les idées reçues (du type « la violence dans les médias pousse les jeunes à être délinquants »), et envisager les individus non pas seulement comme des récepteurs de messages médiatiques mais aussi comme des acteurs culturels. Ce qui implique de déplacer les questionnements du «What?» [Quoi ?] (e.g. que pensez-vous de...?) au «Why?» [Pourquoi ?] (e.g. pourquoi pensez-vous que...?). On étudie ainsi davantage des processus de réception, d'attribution de signification, des actes d'interprétation, plutôt que de vérifier si la communication est bien passée (p.13).

6 À travers cet ouvrage, l'auteur réaffirme l'intérêt d'étudier les publics, dans une perspective critique inscrite dans le courant des Cultural Studies. Ce,après avoir constaté à quel point il est important pour les acteurs sociaux de connaître la manière dont leurs messages sont reçus (voir l'enquête réalisée par Andy Ruddock sur la réception par des jeunes d'un programme de sensibilisation mis en place par les sapeurs-pompiers britanniques, chapitre 7, pp.156-166). On ne parle donc pas exclusivement de publics médiatiques ici, ce qui suscite un questionnement: dans le programme de recherche proposé, il n'est pas fait mention des éventuelles spécificités de chaque institution médiatique/productrice de messages. L'auteur insiste sur l'intérêt à accorder aux contextes- celui de la réception, celui de la mise en œuvre de l'enquête-, qu'en est-il du contexte de production du message? Ce dernier pourrait ajouter à la liste des influences qui encadrent la réception, puisque les produits des institutions culturelles et médiatiques se situent également au cœur de logiques d'acteurs. 


\section{AUTEURS}

CÉLINE SÉGUR

CREM, université Nancy 2

cesegur@yahoo.fr 